Forum $2017 \cdot 32: 497$

https://doi.org/10.1007/s12312-017-0356-1

Online publiziert: 7. November 2017

(c) Springer Medizin Verlag GmbH 2017 \section{DEUTSCHE
STUDIENGRUPPE

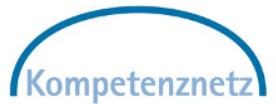 \\ Maligne Lymphome}

Anlässlich ihres 30. Arbeitstreffens am 20./21. Oktober 2017 in Schloss Hohenkammer bei München hat die Deutsche CLL Studiengruppe (DCLLSG) gemeinsam mit dem Kompetenznetz Maligne Lymphome e. V. (KML) eine DIN-A4-Karte zu den aktuellen Behandlungspfaden für Patienten mit einer chronischen lymphatischen Leukämie (CLL) herausgegeben. Abhängig vom Erkrankungsstadium, der Fitness und dem Risikoprofil des Patienten wird grafisch aufbereitet, wie Patienten im Falle einer Ersterkrankung (Firstline-Therapien) oder nach einem Rückfall (Rezidiv-Therapien) behandelt werden sollten - vorzugsweise im Rahmen von DCLLSG-Studien oder nach einem von der DCLLSG empfohlenen Standard.

Die Deutsche CLL Studiengruppe (DCLLSG) unter der Leitung von Prof. Dr. Michael Hallek (Universität zu Köln) hat im Rahmen zahlreicher Studien der Phasen I, II und III neue, international anerkannte Standards für die Behandlung von Patienten verschiedener Stadien und Risikoprofile einer CLL etabliert und konnte maßgeblich dazu beitragen, dass sich die Lebenserwartung von CLL-Patienten in den letzten Jahren erheblich verlängert hat. Ein wesentliches Anliegen der Studiengruppe ist es, die Behandlung von CLL-Patienten mit möglichst allen Zentren im deutschsprachigen Raum abzustimmen und sich so zu vernetzen, dass die neuesten Diagnostik- und Therapieoptionen allen CLL-Patienten zur Verfügung gestellt werden können. Dazu tragen vor allem die Studien der DCLLSG bei, wie auch das bislang sehr erfolgreiche DCLLSG-Register, für das alle Behandlungszentren ihre Patienten melden können.

Kompetenznetz Maligne Lymphome e.V. \& Deutsche CLL Studiengruppe

\title{
Aktuelle Therapiepfade und Studienübersicht zur CLL
}

\section{Behandlungsoptionen für CLL-Patienten innerhalb und außerhalb von Studien}

Mit der jetzt veröffentlichten Übersicht können die behandelnden Ärzte auf einen Blick erkennen, ob für ihren jeweiligen CLL-Patienten eine klinische Therapiestudie zur Verfügung steht. Zudem können sie das jeweilige Studienkonzept mit dem empfohlenen DCLLSG-Behandlungsstandard außerhalb von Studien vergleichen. Die als „DCLLSG-Standard“ deklarierten Behandlungspfade stimmen im Wesentlichen mit den DGHO-Leitlinien zur CLL überein, setzen aber aufgrund ihrer Aktualität geringfügig auch andere Akzente.

\section{Behandlungspfade als Studienkarte}

Die Karte mit den DCLLSG-Behandlungspfaden wird regelmäßig aktualisiert und steht auf den Internetseiten der Deutschen CLL Studiengruppe (www.dcllsg.de) und des Kompetenznetzes Maligne Lymphome e.V. (www.lymphome.de) zum Download zur Verfügung (derzeitiger Aktualisierungsstand: 13. Oktober 2017). Behandlungszentren können einzelne Exemplare der laminierten Karte beim KML anfordern.

\section{Chronische lymphatische Leukämie}

Die chronische lymphatische Leukämie, abgekürzt CLL, ist die häufigste Leukämie in westlichen Ländern, an der überwiegend ältere Menschen erkranken. Bei einer CLL werden im Blut, aber auch in den Lymphknoten, der Milz, der Leber und im Knochenmark auffallend viele bösartig veränderte Lymphozyten des Typs B gefunden. Aufgrund der zellulären
Herkunft der Tumorzellen ist die CLL eine Unterform der mehr als 60 verschiedenen Lymphomerkrankungen. Kennzeichnend für die CLL ist ihr eher langsames, schleichendes Voranschreiten. Dennoch zeigen sich abhängig vom Risikoprofil des einzelnen Patienten ganz unterschiedliche klinische Verläufe. Weitere Informationen zur CLL bieten auch die Internetseiten www. lymphome.de und www.dcllsg.de.

\section{Arbeitstreffen der Deutschen CLL Studiengruppe}

Die Arbeitstreffen der DCLLSG finden ein- bis zweimal im Jahr statt. Das Programm des diesjährigen Arbeitstreffens umfasste die Updates aller laufenden Studien, einen Überblick über die geplanten neuen Studien und Informationen über die erhobenen Daten von nicht mehr rekrutierenden Studien. Weitere Informationen: www.dcllsg.de

\section{Korrespondenzadressen}

Kompetenznetz Maligne Lymphome e.V.

KML | Information \& Kommunikation

Uniklinik Köln, 50924 Köln, Deutschland

Telefon +49 (0)221 478-96005

Fax: +49 (0)221 478-96001

silke.hellmich@uk-koeln.de

www.lymphome.de

\section{Deutsche CLL Studiengruppe (DCLLSG)}

Klinik I für Innere Medizin

Uniklinik Köln, 50924 Köln, Deutschland

Telefon: +49 (0)221 478-88220

Fax: +49 (0)221 478-86886

cllstudie@uk-koeln.de

www.dcllsg.de 